\title{
Reduction of peak to average power ratio in coherent optical orthogonal frequency division multiplexing using companding transform
}

\begin{abstract}
In this paper, a comparison between the original and companded signal implemented in Coherent Optical OFDM (CO OFDM) is presented. The companded signal based on $\varepsilon$-law algorithm was applied at the transmitter to reduce the Peak to Average Power Ratio (PAPR) by enlarging small signals and compressing large signals. From the results, the companded signal shows 3dB PAPR reduction.
\end{abstract}

Keyword: Algorithm; CO-OFDM; Companding; PAPR; Transmitter 